\title{
Ghemistry of the Active Site of Rhodopsin
}

\author{
By M. Akhtar and M. D. Hirtenstein \\ Department of Physiology and Biochemistry, University of Southampton, SO9 $5 \mathrm{NH}$
}

(Received 12 September 1969)

Rhodopsin, the light-sensitive protein of bovine retina, consists of a lipoprotein, opsin, and a polyene aldehyde, 11-cis-retinal (Krinsky, 1958; Matthews, Hubbard, Brown \& Wald, 1963). Irradiation of rhodopsin with visible light results in the formation of all-trans-retinal and opsin through a number of unstable intermediates, rhodopsin $\left(\lambda_{\max } 498 \mathrm{~nm}\right.$.) $\rightarrow$ pre-lumirhodopsin $\left(\lambda_{\max } .543 \mathrm{~nm}\right.$.) $\rightarrow$ lumirhodopsin $\left(\lambda_{\max } 497 \mathrm{~nm}\right.$.) $\rightarrow$ metarhodopsin I $\left(\lambda_{\max }\right.$. $478 \mathrm{~nm}$. $) \rightarrow$ metarhodopsin II $\left(\lambda_{\max } .380 \mathrm{~nm}\right.$. $) \rightarrow$ all-trans-retinal $\left(\lambda_{\max } .387 \mathrm{~nm}\right.$. $)+$ opsin, which possess characteristic absorption spectra (Matthews et al. 1963). Although rhodopsin itself is resistant to treatment with sodium borohydride, it was shown that irradiation of rhodopsin solution in the presence of sodium borohydride resulted in the formation of a reduced derivative of rhodopsin (Akhtar, Blosse \& Dewhurst, 1965; Bownds \& Wald, 1965). The new derivative was formulated as dihydrometarhodopsin II (Akhtar, Blosse \& Dewhurst, 1965, 1967, 1968). Structural studies carried out on dihydrometarhodopsin II revealed that the derivative contained the retinyl moiety attached to the protein through an e-amino group of lysine (Akhtar et al. 1967, 1968). Parallel results were reported by Bownds (1967). The question, however, remained unanswered whether or not the retinal-opsin linkage identified above was also present in the native rhodopsin. A suggestion was made as early as 1958 that native rhodopsin may contain the polyene aldehyde moiety linked to a phospholipid component (Krinsky, 1958; see also Bonting \& Bangham, 1967).

Kito, Suzuki, Azuma \& Sekoguti (1968) noted that the treatment of rhodopsin $\left(\lambda_{\max } .500 \mathrm{~nm}\right.$.) with trichloroacetic acid in the dark resulted in the formation of a new derivative ( $\lambda_{\max } 440 \mathrm{~nm}$.). When the latter derivative $\left(\lambda_{\max } 440 \mathrm{~nm}\right.$.) was frozen to $90^{\circ} \mathrm{K}$, the rhodopsin-like spectrum ( $\lambda_{\max } .495 \mathrm{~nm}$.) was regenerated. This spectrum was sensitive to light. The regeneration of a rhodopsin like-species may be rationalized by assuming that acid-denatured rhodopsin contained retinal in the 11-cis configuration and still linked to the native site. With the information at hand the problem had been simplified, and our task was therefore to identify the site of attachment of the retinyl moiety in the derivative of rhodopsin produced by trichloroacetic acid.
In this paper the stabilization of the derivative with $\lambda_{\max } .440 \mathrm{~nm}$. with sodium borohydride and the subsequent extraction with methanol of a phospholipid-linked retinyl moiety are described. A quantitative evaluation of the problem was made possible by using radioactive rhodopsin labelled specifically at the active site. The problem of the nature of the retinal-opsin linkage in the native rhodopsin was approached by Poincelot, Millar, Kimbel \& Abrahamson (1969), who used techniques and assumptions different from those used in the present work.

Tritiated rhodopsin containing label in the retinyl moiety was prepared essentially as described previously (Akhtar et al. 1968), except that tritiated 11-cis-retinal was further purified by rechromatography (Akhtar et al. 1968). This purification was necessary because the projected investigation required rhodopsin of high radiochemical purity and particularly free from unbound radioactive contamination. The rhodopsin solution $(3 \mathrm{ml}$., with $E_{500}^{1 \mathrm{~cm}} \cdot 0.8$ and containing $5 \times 10^{4}$ c.p.m.) was treated with trichloroacetic acid ( $0 \cdot 1 \mathrm{M}$ final concentration). The resulting solution $\left(\lambda_{\max } 440 \mathrm{~nm}\right.$.; $\left.\mathrm{pH} 2 \cdot 8\right)$ on treatment with sodium borohydride $(15 \mathrm{mg}$.) gave a new derivative having $\lambda_{\max } .333 \mathrm{~nm}$. and suggesting that the acid-denatured rhodopsin contained a group, presumably $>\mathrm{C}=\mathrm{N}-$, that could be reduced. When this solution was freezedried about $60 \%$ of the original radioactivity was extractable into methanol and the remaining $40 \%$ of the radioactivity was associated with the methanol-insoluble protein fraction. On the other hand, however, when an identical sample of rhodopsin was irradiated in the presence of sodium borohydride, to give dihydrometarhodopsin II, and freeze-dried less than $10 \%$ of the original radioactivity was soluble in methanol; the remaining $90 \%$, as expected, remained bound to the protein precipitate (Akhtar et al. 1968).

The methanol extract from the reduced aciddenatured rhodopsin was analysed by t.l.c. with chloroform-methanol $(75: 11, \mathrm{v} / \mathrm{v})$. In this solvent system at least $70 \%$ of the radioactivity was confined to a band that had an $R_{F}$ value $(0.43)$ identical with that of synthetic $N$-retinylphosphatidylethanolamine. The remaining radioactivity was at the solvent front (less than $10 \%$ ), at $R_{F} 0.70$ (less 


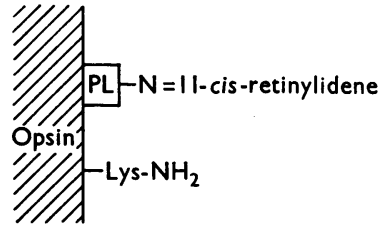

Rhodopsin (I)

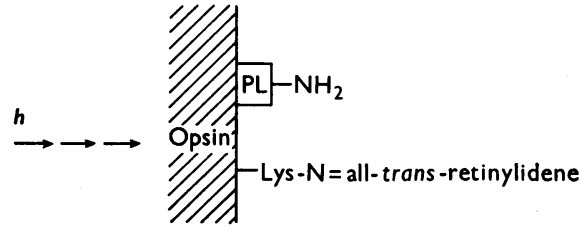

Metarhodopsin II

(II)

Scheme 1. PL, Phospholipid.

than $10 \%$; retinol) and $R_{F} 0.25$ (less than $10 \%$; unknown). In this solvent system $N$-retinylphosphatidylserine had $R_{F} \mathbf{0 \cdot 3 6}$.

The derivative with $\lambda_{\max }$. 440nm. prepared by denaturation with trichloroacetic acid had previously been shown to be converted into a rhodopsin-like species by freezing (Kito et al. 1968). In the present work this derivative $\left(\lambda_{\max } 440 \mathrm{~nm}\right.$.) was stabilized with sodium borohydride, and a phospholipid-linked retinyl derivative containing at least $42 \%$ (based on the t.l.c. radioactivity) of the original radioactivity was extracted with methanol. That in this experiment $40 \%$ of the original radioactivity was also associated with the protein fraction forbids an unambiguous interpretation of the results. However, these observations highlight the fact that a lipid amino group may play an important role in the active site of rhodopsin. This supports the inspired suggestion originally made by Krinsky (1958) and forcefully endorsed by Poincelot et al. (1969). Since the retinyl moiety at the metarhodopsin II stage is exclusively linked to the protein component of opsin, the overall process of bleaching of rhodopsin probably involves, not only the isomerization of the 11-cis-retinal to the alltrans configuration, but also the migration of the retinyl moiety from one amino group to another as shown in Scheme 1.

It was suggested that the $\lambda_{\max }$. of rhodopsin is due to an intramolecular charge-transfer interaction between the $N$-retinylidene chromophore and a suitably located group $-\mathrm{X}$ or $-\mathrm{X}-\mathrm{H}$ of opsin. The spectral change in the conversion of rhodopsin (I) into metarhodopsin II (II) was attributed to the dislocation of the interaction (Akhtar et al. 1965, 1968). The new evidence, indicating the shift of the retinyl moiety in this conversion, offers a direct explanation for the dislocation of the two groups.

Materials and methods. Phosphatidylethanolamine and phosphatidylserine were obtained from L. Light and Co. Ltd., Colnbrook, Bucks. All other chemicals were obtained as described previously (Akhtar et al. 1968). 11-cis-Retinal, tritiated 11-cis-retinal, rhodopsin, opsin and tritiated rhodopsin were prepared; radioactivity measurements were made and preparative and analytical t.l.c. was performed as described previously (Akhtar et al. 1968). $N$-Retinylphospholipid derivatives were made by the method previously used for the preparation of similar derivatives of amino acids (Akhtar et al. 1968).

M.D.H. thanks the Science Research Council for a research studentship and $M$. A. thanks them for a research grant. We extend our thanks to the F. M. C. Meat Corporation (Salisbury) for their co-operation in the collection of fresh eyes. We thank Professor K. A. Munday for his kind interest and encouragement.

Akhtar, M., Blosse, P. T. \& Dewhurst, P. B. (1965). Life Sci. 4, 1221.

Akhtar, M., Blosse, P. T. \& Dewhurst, P. B. (1967). Chem. Commun. p. 631.

Akhtar, M., Blosse, P. T. \& Dewhurst, P. B. (1968). Biochem.J.110, 693.

Bonting, S. L. \& Bangham, A. D. (1967). Exp. Eye Res. 6, 400.

Bownds, D. (1967). Nature, Lond., 216, 1178.

Bownds, D. \& Wald, G. (1965). Nature, Lond., 205, 254.

Kito, Y., Suzuki, T., Azuma, M. \& Sekoguti, Y. (1968). Nature, Lond., 218, 955.

Krinsky, N. I. (1958). Arch. Ophthalmol. 60, 688.

Matthews, R. G., Hubbard, R., Brown, P. K. \& Wald, G. (1963). J. gen. Physiol. 47, 215.

Poincelot, R. P., Millar, P. G., Kimbel, R. L. \& Abrahamson, E. W. (1969). Nature, Lond., 221, 256. 